Session 3557

\title{
INTEGRATING RISK INTO AN ENGINEERING ECONOMY COURSE WITH SIMULATION SOFTWARE
}

\author{
Eyler R. Coates, Rita L. Endt \\ The University of Southern Mississippi
}

\begin{abstract}
Engineering economy problems with all deterministic inputs are actually rare. Some information required for solving engineering economy problems can be defined fairly well, but much information is uncertain, such as the actual cash flows from revenues and costs, the salvage value of equipment, the interest rate or even the project life. The use of simulation software with Monte Carlo techniques makes engineering economy problem solutions more realistic. Probability descriptions of input variables and Monte Carlo sampling together provide a practical method of finding the distribution of the desired output given the various random and deterministic input variables. The results of such analyses give better information for making decisions.
\end{abstract}

This paper provides two examples that demonstrate how commonly available simulation software could be used in engineering economy problems to reduce the risk associated with the solutions. One example demonstrates the future worth distribution of an annual series of payments when there is uncertainty about the future earning power (interest rate) from year to year. Also, another example extends the first example to include an input variable that is dependent upon another random input variable. The second example also demonstrates how a discontinuous function can be easily incorporated in the economic model using simulation. These examples can be used to demonstrate how risk is handled in an engineering economy course. The examples can also be used as additional applications in an industrial simulation course.

Introduction

Some information required for an engineering economic problem can be well defined, like the cost of new machinery, labor rates or the current tax rate structure. Much required information is uncertain, such as the actual cash flows from revenues and costs, the salvage value of equipment, the interest rate or even the project life. In most undergraduate engineering economy courses, the concept of risk is either not introduced at all or is mentioned briefly at the end of a course and in final chapters of a textbook ${ }^{5}$. Yet, engineering economy problems with all deterministic inputs are actually rare in "real life." 
There are a number of approaches for handling economic risk that are used today. One approach is scenario analysis. However, as Park has pointed out, worst-case and best-case scenarios are not easy to interpret and do not provide probabilities of occurrence of those possibilities nor do they normally provide additional information such as the probability of losing money on a project or the probability of other possibilities ${ }^{9}$. Sensitivity analysis and spider plots can provide insight into a engineering economy problems but are not appropriate when there is statistical dependence between variables ${ }^{3}$. Probability descriptions of input variables allow further refinement of the analysis of economic risk and allow the output of a distribution for the desired answer.

The notion of probability distributions to describe economic risk has been around for many years before the advent of computers and advanced software technology. Hillier, in his groundbreaking paper in 1963, proposed the use of probability distributions of present worth to properly convey project risk information to augment other methods such as expected value of the present worth and sensitivity analysis of individual inputs ${ }^{6}$. He also demonstrated that the probability distribution of present worth, or annual worth or internal rate of return can under certain assumptions be derived from yearly cash flows that are themselves random variables. He presented equations for the net present worth (NPV) distribution parameters when the cash flows were mutually independent random variables and when the cash flows were random but perfectly correlated to each other. Later, Giaccotto derived the distribution parameters for the NPV when the cash flows were correlated by a first order autoregressive stochastic process (or a Markov process) ${ }^{4}$. As projects become complicated, derivations of the probability distribution of the NPV as a function of the unknown random input variables can be tedious or impossible ${ }^{10}$.

Monte Carlo sampling provides a practical method of finding the distribution of the NPV or future worth from the various random input variables. Coats and Chesser showed that Monte Carlo techniques could be used in the corporate financial model to produce associated probabilities of occurrence, confidence intervals and standard deviations in addition to standard financial reports ${ }^{2}$. Seila and Banks showed that an electronic spreadsheet could be used to simulate project risk with Monte Carlo techniques ${ }^{10}$. The methods for risk analysis of projects have been published for a long time and the availability of computers and software is pervasive. Ho and Pike report that "proponents of risk analysis argue that increased risk information improves management's understanding of the nature of risks, helps identify the major threats to project profitability and reduces forecasting errors ${ }^{7}$." They also report that "the risk analysis approach provides useful insights into the project, improves decision quality and increases decision confidence ${ }^{7}$." But, the risk analysis approach is one aspect of economic analyses that is commonly ignored during project evaluations. Typically, deterministic data is "forced" even when there is uncertainty. The values are usually random, but are made to be point estimates without regard to the risk or sensitivity of error induced by assuming point estimates. Therefore, the outcome of the analysis has a greater probability of being wrong. A good project could be rejected or a bad project approved erroneously. Including risk analyses in engineering economy solutions is an important step to achieving more accurate information to make better decisions. Simulations with Monte Carlo techniques is one way to do risk analysis in engineering economy solutions. 
Below is a chart that illustrates the differences between Hillier's approach and a simulation approach for solving engineering economy problems.

Hillier's Approach

- Variable cash flows; year to year flows are independent

- theoretical distributions which are independent

- end of year conventions $\underline{\text { Simulation Approach }}$

- cash flows can be independent or dependent

- cash flows can be theoretical or empirical data

- timing of cash flows can be at any point

- input variables can be correlated with other variables or auto-correlated

- discontinuities are possible

Risk analysis approaches are covered in a few college class curriculums, but never fully to the extent that is necessary for students to be competent in industry. Risk analysis has been typically ignored not just because of the lack of knowledge but also because in the past it was cumbersome and difficult to implement or use. It was very time consuming and any software available was very expensive and needed large computers systems. Then, with the advent of personal computers (PC), spreadsheet capabilities, cheaper simulation software, and ease of use, performing risk analysis is easy. Numbers can be "crunched" and many simulation replications can be done in a small amount of time on relatively inexpensive computers.

Given that the capabilities of doing risk analyses are available, Goyal et al. has questioned why there is so little exposure to the stochastic nature of project cash flows and other project variables in undergraduate curricula ${ }^{5}$. Many in industry know that risk analyses exist, but few can apply the concepts to practice. Therefore, it would greatly enhance the worth of today's engineering students if they would be capable of performing such analyses in industry.

Computer simulation using Monte Carlo techniques has been a part of the industrial engineering and management science curriculums for many years. Fortunately, simulation packages that are already available to students are particularly well suited for sampling from various theoretical and data-defined statistical distributions. In addition, these simulation packages can handle large amounts of sampling data and they have good output reporting capabilities. Thus, students have access to off-the-shelf simulation packages either through their school or through student versions of professional simulation packages that often come with their textbooks. As these students become professionals, they would be able to access these same simulation packages. The next section will demonstrate the ease that engineering economy problems with stochastic input variables can be simulated with industrial simulation software.

\section{Problem 1: A Future Worth Problem With Uncertain Growth Rates}

One typical class of engineering economy problems involves calculating the future worth of a series of cash flows. As an example, suppose that we want to save $\$ 10,000$ per year for the next 
10 years. We would naturally be interested in how much worth would be accumulated at the end. If the growth rate was fixed and known, then the calculation of the future worth would be straightforward. However, the authors feel that it is important to let the students be aware that in the real world there are many investment possibilities where the growth rate is variable from year to year.

Suppose that the investment vehicle chosen was an S\&P 500 index fund. The annual returns from 1954 till 1993 given by Bernstein indicate that the arithmetic average annual return was $13.1 \%$ and the overall equivalent annual return over the entire 40 years was $11.75 \%{ }^{1}$. However, the individual annual returns can vary from $-26.5 \%$ to $52.6 \%$. This variability of annual returns and their timing of the more extreme values can create substantially different final results. The standard future worth formula ignores this variability and can only give a point estimate. Using simulation with Monte Carlo techniques to sample the annual return distribution to generate the multiple possible outcomes would better represent the actual economic situation.

Simulation software such as Arena could be used to estimate the future worth distribution. The following steps describe a method of solving the problem:

1. Determine the input variables

2. Create a flowchart for a single scenario

3. Identify the entity for the simulation

4. Determine the appropriate attributes that are required for the final result

5. Design and enter the network diagram to generate multiple scenarios according to the flowchart

6. Run the simulation

7. Analyze the output

Each of the above steps are described below:

Step 1 - Determine input variables

In this problem, the only variable that is uncertain is the annual growth rate. The other inputs are the $\$ 10,000$ cash series and the 10 year project life. Both are deterministic data in this problem.

Before designing the simulation logic, there is a need to know whether the random returns are independent from each other, dependent on another input variable or auto-correlated (dependent within itself). A good approach to determine the type of variable is to have the students first plot the returns as shown in Figure 1.

Since students have access to spreadsheets and statistical software, they could also plot the correlation values of the different time lags of the annual returns as shown in Figure 2. In this case, the students would see from Figure 2 that the annual returns are essentially independent from year to year. 


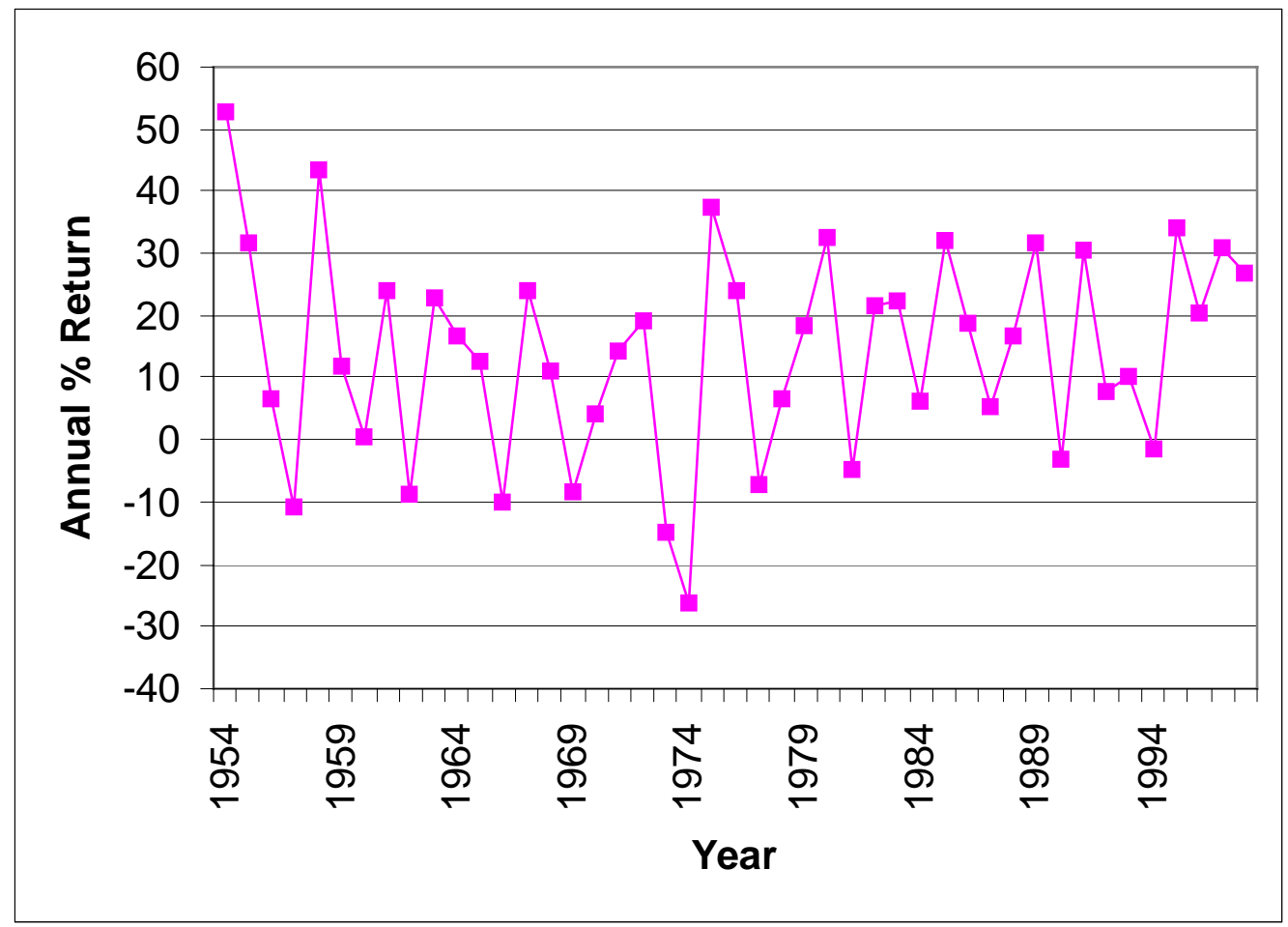

Fig. 1. Time Plot of Annual Returns for the S\&P 500 Index

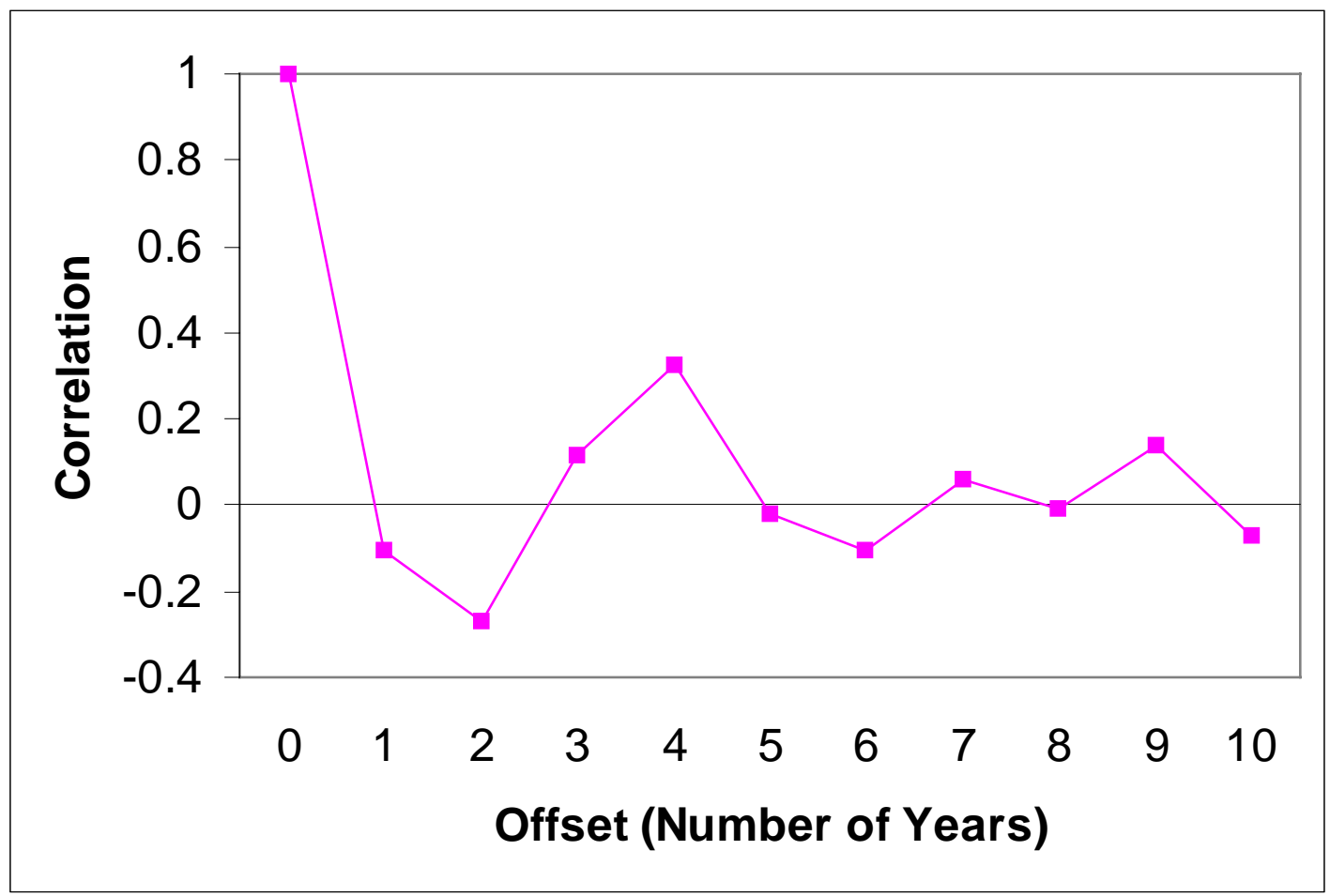

Fig. 2. Correlation of Annual Returns over Time 
A simulation software package such as Arena can allow historical data to be used directly in the simulation or it can automate the process of fitting a probability distribution to the data. If a probability distribution is fitted to the data, Arena allows the user to select an appropriate distribution or Arena can select the closest fitting distribution itself ${ }^{8}$. For the S\&P 500 annual returns, Arena chose the normal distribution with a mean of $13.1 \%$ and a standard deviation of $16.9 \%$ as the best fitting distribution. Unless there was a theoretical reason that called for a particular distribution, a good approach is to allow Arena to choose the best fit distribution. The Arena choice of the normal distribution as best fit agrees with Bernstein ${ }^{1}$. The fit is shown in Figure 3.

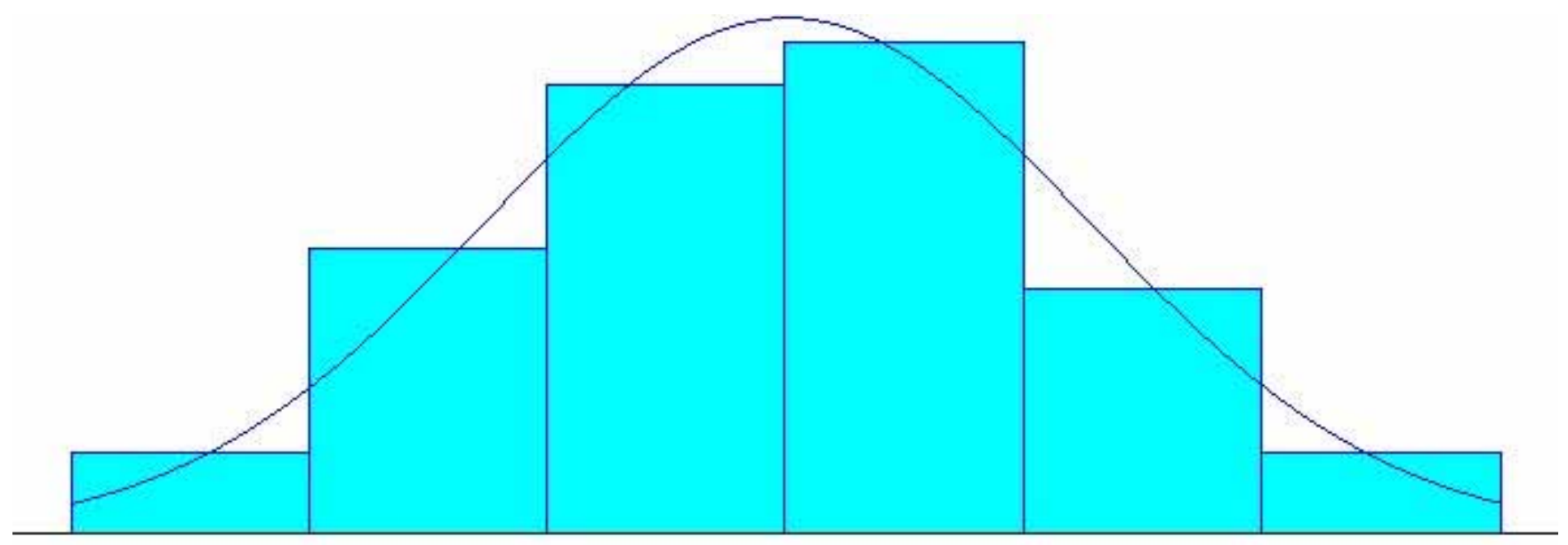

Fig. 3 Fit of Normal Curve to Historical S\&P 500 Index Annual Returns

Because the annual returns are independent and normally distributed, this simplifies the problem of generating random variables for the simulation.

Step 2 - Create flowchart

The student needs to create the flowchart for calculating an individual scenario. The flowchart for this problem is shown in Figure 4. The flowchart assumes an investment made at the end of the year as is the convention in most engineering economic textbooks. Once the student understands the basic steps to generate a single scenario, the transition to a simulation flowchart is rather simple.

Step 3 - Identify entity

Identify the entity in the simulation to be the $\$ 10,000$ annual cash investment over 10 years. Each entity, in the simulation run, will represent a replication (scenario) of the future worth calculation. After an entity is created, the annual growth rates (interest rates) are selected via sampling from a selected distribution. 


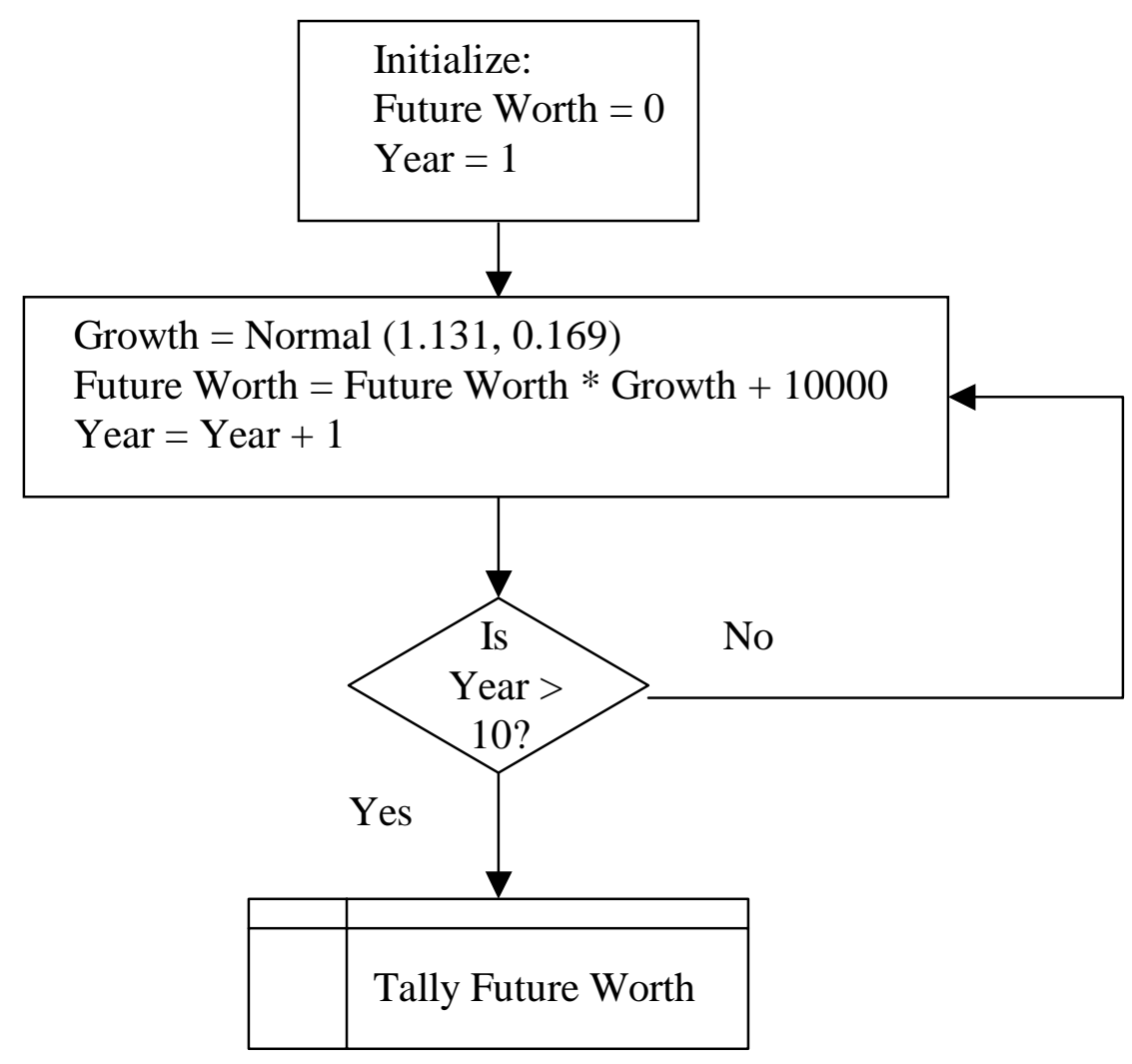

Fig. 4 Flowchart of a Future Worth Calculation for a Given Scenario

Step 4 - Determine attributes

Three variables are needed in order to calculate the future worth iteratively. From the flowchart in Figure 4, the three variables required are an accumulator (future worth), a counter (year) and the random variable which is the annual return (growth).

Step 5 - Design and enter the network diagram

The Arena network diagram to simulate the problem is given in Fig. 5. Note that the simulation network is rather elegant and well within the grasp of the average student. There is almost a oneto-one relationship between the Arena simulation network diagram in Figure 5 and the flowchart given in Figure 4. The Arrive module allows for the creation of entities. Each entity represents one scenario. The Arrive module also allows the attributes Year and Future Worth to be initialized. The Actions Module makes the iterative assignments to the attributes Growth, Future Worth and Year. The Branch module serves the function of the decision node. The Depart module allows the attribute Future Worth to be tallied before the entity is destroyed. The Arena network requires two additional modules. The Simulate module is required only to enter the title of the report and the name of the analyst. The Statistics module is required to tell the simulation software to save the output data into a file for later analysis. There are several methods for 
stopping the simulation. The method chosen was to limit the number of created entities in the Arrive module. For this example, the number of entities (scenarios) was 5000.

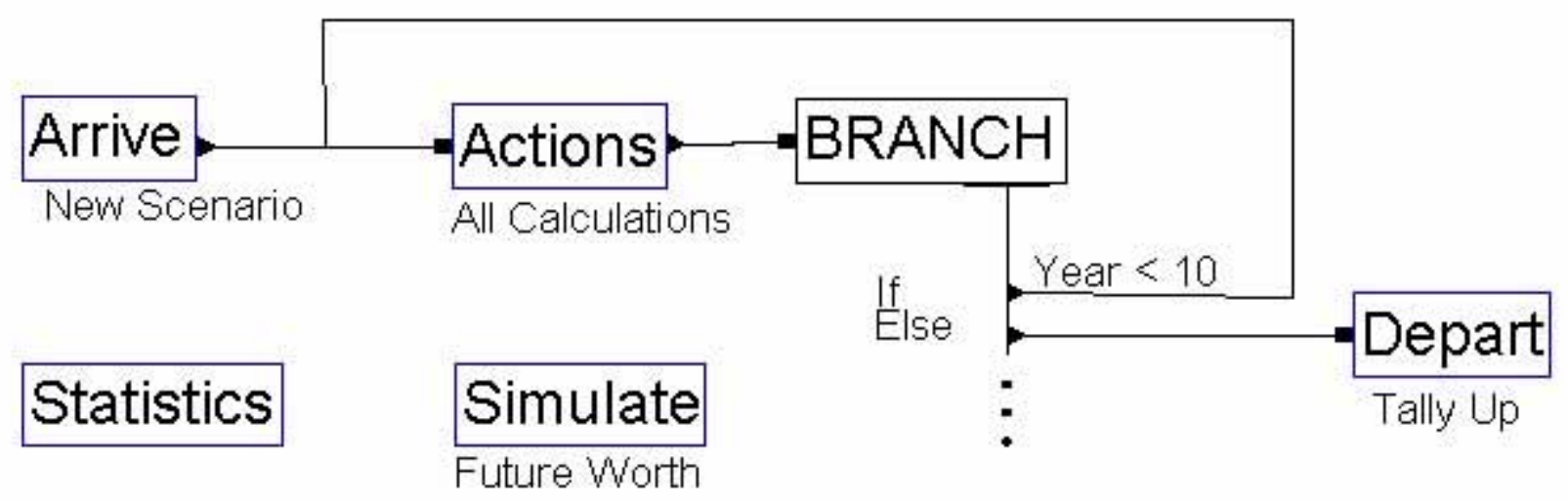

Fig.5. Simulation Network Diagram For Future Worth Estimation with Variable Interest Rates

Step 6 - Run the simulation

The future worth associated with the particular scenario (entity) is calculated year by year with a new sampled growth rate for each year. This future worth is stored as an attribute of the entity. Before the entity leaves the system, the future worth is collected as a statistic and tabulated by the simulation software.

Step 7 - Analyze the output

The output of the simulation program is given in Fig. 6. Because each entity was created one time unit apart, the current time on the Arena report also reflects the number of replications in the study (5000). The output reporting capabilities of simulation packages are used to advantage here. Summary statistics are automatically generated. The student can see that the future worth of the 5000 scenarios ranges from $\$ 61,366$ to $\$ 397,660$. Based on the histogram, the student can determine any prediction (tolerance) interval. For example, it appears that $90 \%$ of the observations fall between $\$ 90,000$ to $\$ 230,000$. This means there is a $90 \%$ confidence that the future worth of this series falls between $\$ 90,000$ and $\$ 230,000$. From the text output, the student can obtain the mean expected value of $\$ 154,280$. Incidentally, if we had used the standard equal payment series future worth calculation with 10 years at $11.75 \%$ fixed annual return, the point estimate would have been $\$ 173,380$ with no indication of the probable range. 
ARENA Simulation Results

Dr. Eyler Robert Coates, Jr. - License \#9910153

Summary for Replication 1 of 1

Project: Future Worth

Analyst: Endt and Coates

Replication ended at time

Identifier

Average

1.5428E+05 1175.7

61366.

$3.9766 \mathrm{E}+05$

5000

COUNTERS

\begin{tabular}{lcc} 
Identifier & Count & Limit \\
\hline Replications & 5000 & Infinite
\end{tabular}

Simulation run time: 0.08 minutes.

Simulation run complete.

Frequency

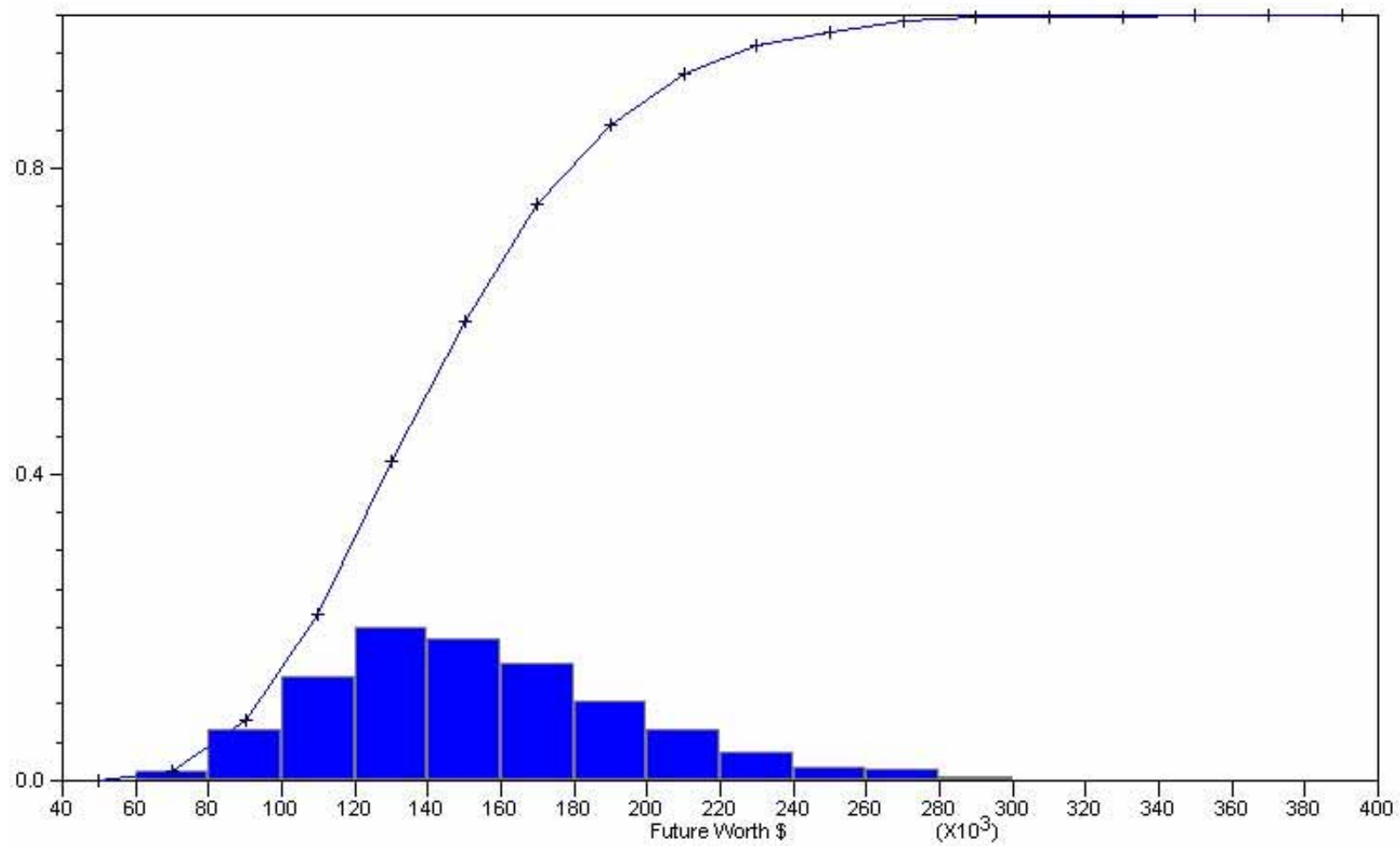

Fig. 6. Simulation Output from Network in Fig. 5 
Problem 2: An Extension of Problem 1 with Dependent Input Variables and Discontinuity

Problem 1 can be extended to include a number of complications. As an example, suppose that the amount that is invested each year is not a constant $\$ 10,000$. Furthermore, suppose that the amount invested is influenced by the current performance of the S\&P 500 index. The approach to the problem would still follow the seven step approach outlined before. Only the changes in each step due to the extensions are given below.

Step 1 - Determine input variables

In addition to the uncertainty in the annual growth rate, suppose that the amount invested each year depends on whether the S\&P500 index is performing well. Rightly or wrongly, an investor may be hesitant to make an investment if the S\&P500 index is declining (The growth rate multiplier, Growth, is less than 1). For this example, let the amount of investment be $\$ 10,000$ if the growth rate of the index is greater than 1 and let the investment be $\$ 0$ if the index is declining (growth rate of less than 1). A binary indicator such as (Growth $>1$ ) would evaluate to 1 if the growth rate factor (defined by Growth) was greater than 1 and the indicator would evaluate to 0 if the growth rate factor was less than 1 . The amount invested each year would be calculated by $\$ 10000 *$ (Growth > 1). This would, in effect, cause the amount invested to be influenced by the current S\&P500 index performance and it would also model a discontinuity in the function of the annual investment amount.

Step 2 - Create flowchart

The student needs to create the flowchart for calculating an individual scenario. The only change in the flowchart would occur in the iterative calculation block. This change is shown below in Figure 7.

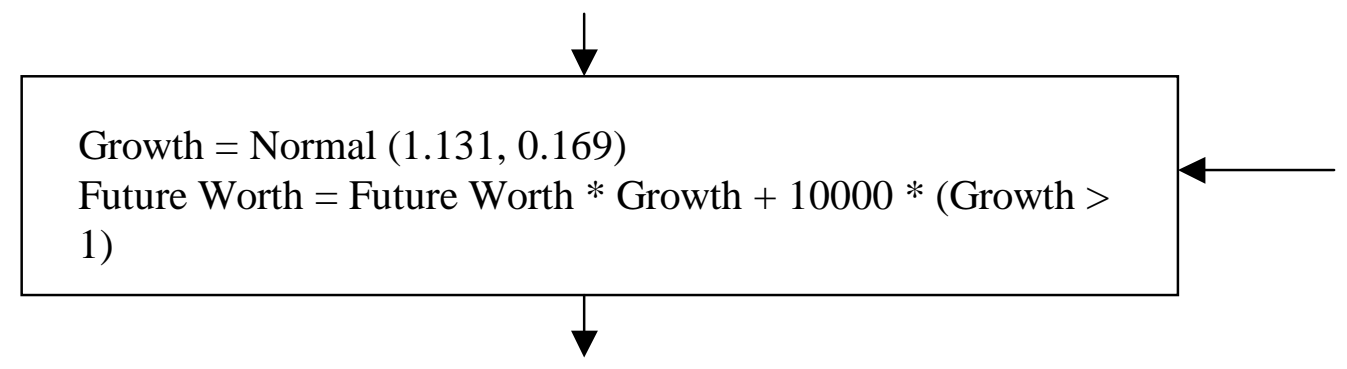

Fig. 7. Adjustment to Flowchart for Dependent Input Variable

Step 3 - Identify entity and Step 4 - Determine attributes

These steps would not change from the previous problem.

Step 5 - Design and enter the network diagram 
In this step, only the Actions module would change to reflect the new calculation required from Figure 7.

Step 6 - Run the simulation

This step would not change from the previous problem.

Step 7 - Analyze the output

The output of the simulation program is given in Fig. 8. The comments parallel the previous problem. The future worth of the 5000 scenarios ranges from $\$ 15,471$ to $\$ 397,660$. Based on the histogram, it appears that $90 \%$ of the observations fall between $\$ 40,000$ to $\$ 210,000$. This means there is a $90 \%$ confidence that the future worth of this series falls between $\$ 40,000$ and $\$ 210,000$. From the text output, the student can obtain the mean expected value of $\$ 120,680$. Incidentally, if we had used the standard equal payment series future worth calculation with 10 years at $11.75 \%$ fixed annual return, the point estimate would have been $\$ 173,380$ with no indication of the probable range. Incorporating the additional complications resulted in a substantially different output from the previous problem.

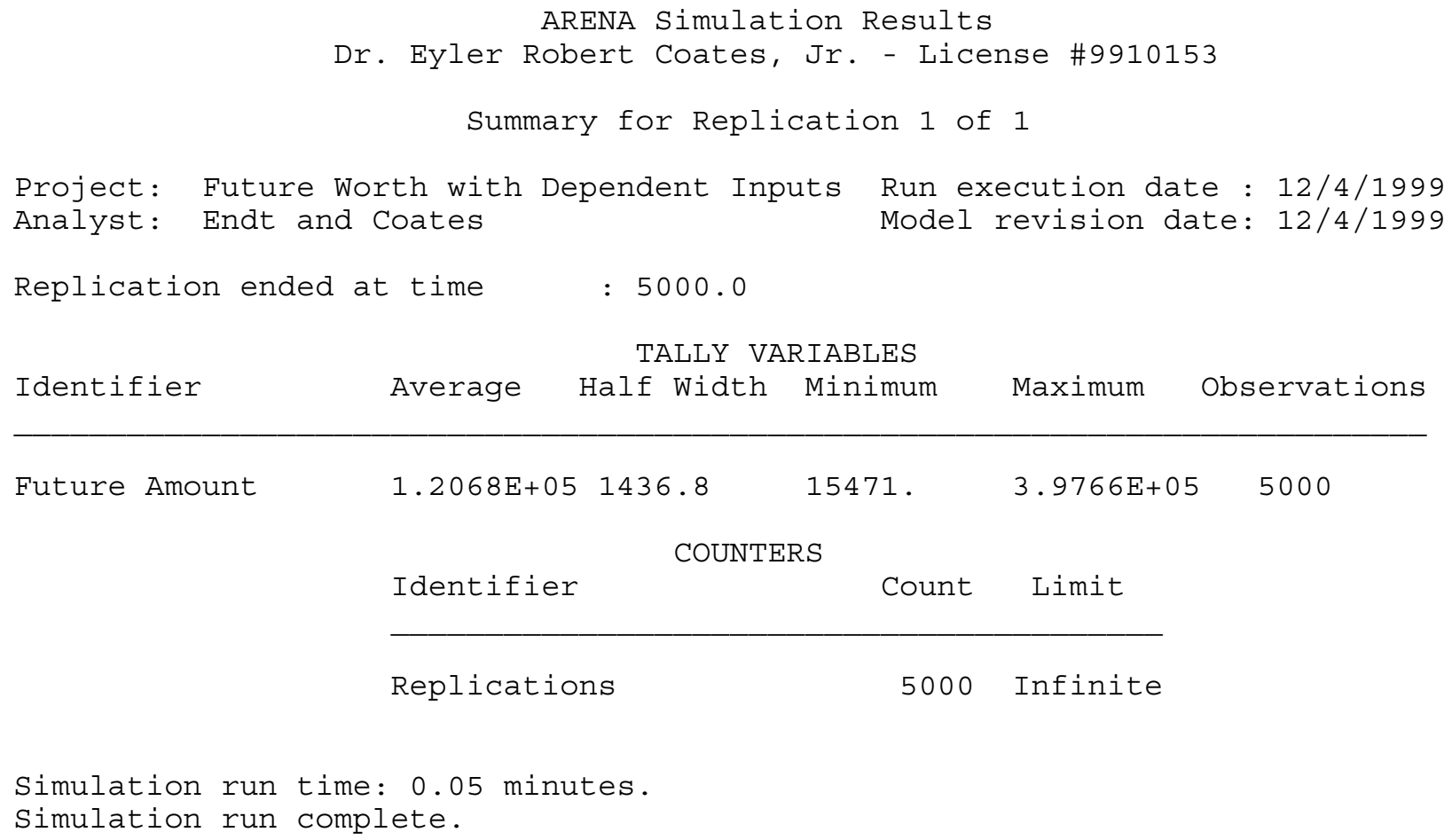




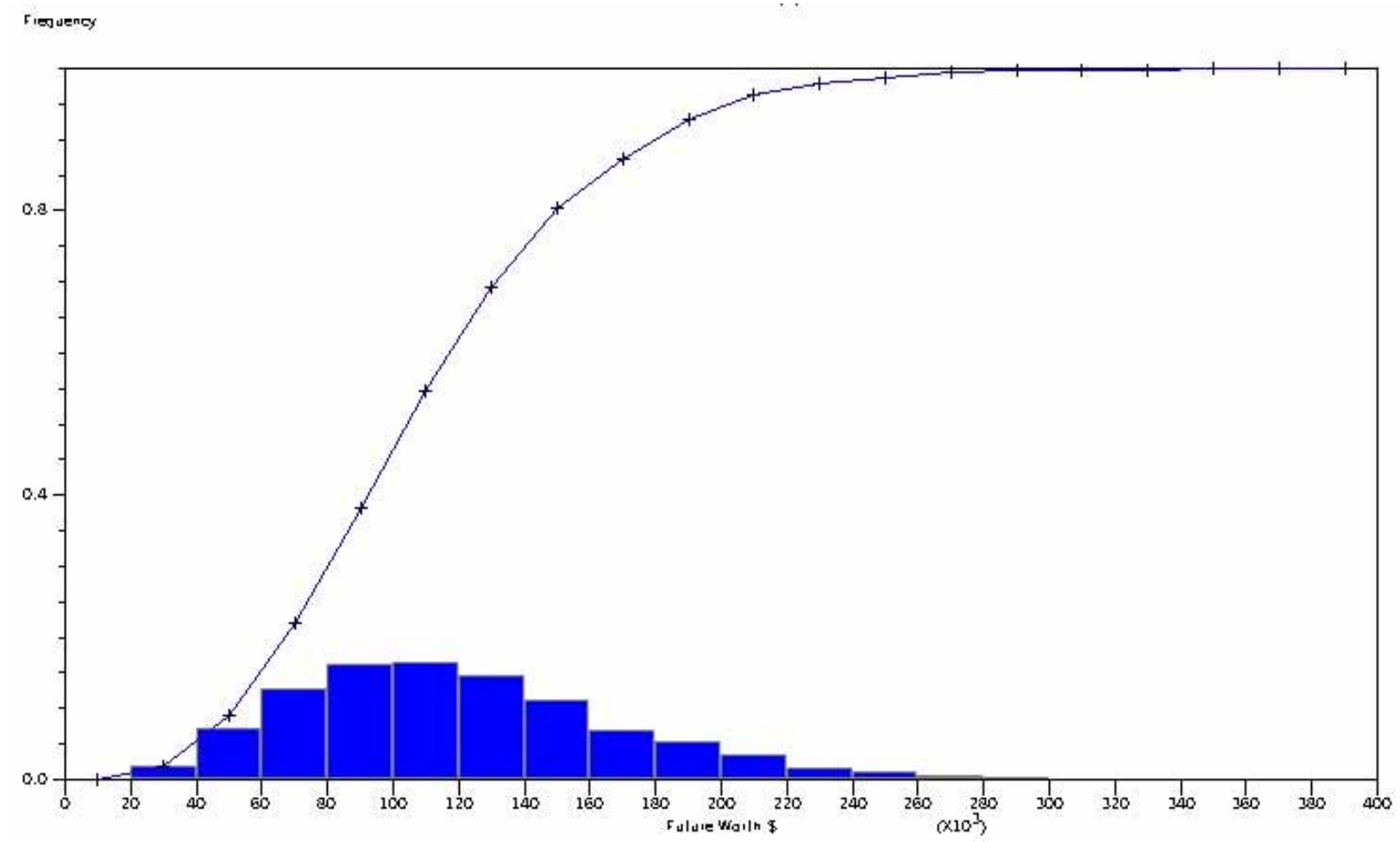

Fig. 8. Simulation Output from Extended Problem

\section{Conclusion}

This paper provided two examples that demonstrate how commonly available simulation software could be used in engineering economy problems. One example generated the distribution future worth of an annual series of payments when there is uncertainty about the future earning power (interest rate) from year to year. The second example demonstrated how complications such as dependent inputs and discontinuities can be easily incorporated using the simulation approach. These examples can be used to demonstrate how risk is handled in an engineering economy course. The examples can also be used as additional novel applications in an industrial simulation course. Hopefully, students who are exposed to these methods at the undergraduate level will carry these practices into the workplace and advance the accuracy of representing risk in engineering economic decision making.

\section{Bibliography}

1. Bernstein, P.L., [1995], The Portable MBA in Investment, John Wiley and Sons, New York, NY.

2. Coats, P.K., and D.L. Chesser, [1982], Coping With Business Risk Through Probabilistic Financial Statements, Simulation, June 1982, 111-121. 
3. Eschenbach, T.G., and R.J. Gimpel, [1990], Stochastic Sensitivity Analysis, The Engineering Economist, 35(4), 305-321.

4. Giaccotto, C., [1984], A Simplified Approach To Risk Analysis In Capital Budgeting With Serially Correlated Cash Flows, The Engineering Economist, 29(4), 273-286.

5. Goyal, A.K., J.M. Tien, and P.A. Voss, [1997], Integrating Uncertainty Considerations In Learning Engineering Economy, The Engineering Economist, 42(3), 249-257.

6. Hillier, F.S., [1963], The Derivation of Probabilistic Information For The Evaluation Of Risky Investments, Management Science, 1963, 443-457.

7. Ho, S.S.M., and R.H. Pike, [1998], Organizational Characteristics Influencing The Use Of Risk Analysis In Strategic Capital Investments, The Engineering Economist, 43(3), 247-268.

8. Kelton, W.D., R.P. Sadowski, and D.A. Sadowski, [1998], Simulation with Arena, WCB/McGraw-Hill, Boston, MA.

9. Park, C.S., [1997], Contemporary Engineering Economics, $2^{\text {nd }}$ Edition, Addison-Wesley, Menlo Park CA.

10. Seila, A.F., and J. Banks, [1990], Spreadsheet Risk Analysis Using Simulation, Simulation, 57, 163-170.

\section{EYLER R. COATES}

Eyler Coates is an Assistant Professor of Engineering Technology at The University of Southern Mississippi in Hattiesburg. He has 12 years of industrial work experience with manufacturers performing industrial engineering functions. He received a B.S. degree in Industrial Engineering (1979), a M.S. degree in Engineering Science (1996), and a Ph.D. in Engineering Science (1998) all from Louisiana State University in Baton Rouge.

\section{RITA L. ENDT}

Rita Endt is an Assistant Professor of Engineering Technology at The University of Southern Mississippi in Hattiesburg. She has over 25 years of industrial experience in industrial engineering, operations research, and data processing for several large corporations such as General Motors Corporation, Lucas CAV, Electronic Data Systems (EDS) and the United States Navy. She has a B.S. degree in Industrial Engineering (1975) and a M.S. degree in Industrial Engineering (1977) from Wayne State University in Detroit, Michigan. 